\title{
On the Design of Exoskeleton Suit: An Interdisciplinary Project Development Platform for Experiential Learning in Engineering Education
}

\section{Dr. Lei Zhang, University of Maryland, Eastern Shore}

Dr. Lei Zhang received his Ph.D. Degree in Electrical Engineering on 2011 from the University of Nevada, Las Vegas. Since 2012 he is working in the Department of Engineering and Aviation Sciences, University of Maryland Eastern Shore. His main research interests include image processing, autonomous system, optical SoC/NoC architecture, and on-chip optoelectronic device design.

\section{Mr. JeanPaul Badjo, Badjo-T Industries}

Electrical Engineering PHD Student Founder and CEO of Badjo-T Industries

\section{Prof. Ibibia K. Dabipi, University of Maryland, Eastern Shore}

I. K. Dabipi has been in academia for approximately 26 years during which he worked for Bellcore, AT\&T Bell Labs and Southern University. He was the Chair of the Electrical Engineering Department at Southern University from 1997 to 2001. He was Professor and Chair of the Engineering and Aviation Science Department at University of Maryland Eastern Shore from 2001 - 2006. He is currently a Professor in the Department of Engineering and Aviation Sciences at University of Maryland Eastern Shore.His research interests are in the areas of Computer Security and Network Management, Parallel Computing and Algorithms Development, Performance Evaluation of Computer Networks, Optimization of Transportation Networks, and Economic Analysis of Transportation Facilities and Human factors in Aviation Security. He is a member of ASEE, HKN, ACM and a senior member of IEEE

\section{Dr. Xianfang Tan}

Dr. Xianfang Tan is an assistant professor of Engineering Technology in the STEM division at Delaware County Community College. Her research interests include computer architecture, network-on-chip (ONoC), photonic/optical NoC etc. 


\title{
On the Design of Exoskeleton Suit - an Interdisciplinary Project Development Platform for Experiential Learning in Engineering Education
}

\begin{abstract}
Many studies have shown the key to promote STEM education stands on motivating and engaging students in studying and improving their learning performance. Experiential learning is proved to be an effective pedagogy in promoting student learning experience, especially in student-centered interest-based activities. In this paper, we present our exploration in applying the experiential learning in engineering education through the design and development of an exoskeleton suit which can be used as a multifunctional platform for various interdisciplinary projects. The platform enables students in practicing diversified engineering design and explore innovative technologies, such as CAD design and 3D printing, microprocessor and microcomputer system design, image processing, sensor and signal processing, wireless communication, etc. Through the development of the suit, participated engineering students are strongly motivated and significantly promoted in engineering learning, practicing, exploring and innovating. The design of the exoskeleton is patented and the leader student developers received awards in different competitions.
\end{abstract}

\section{Keywords}

STEM Education, Exo-Skeleton Suit, Interdisciplinary Engineering Project Platform

\section{Introduction}

The key to promoting STEM education stands on how to motivate and engage students in studying and therefore improving their learning performance [1]. Various pedagogies have been proposed and practiced for this purpose, among which Experiential Learning has been proved to be an effective educational approach to motivate and engage students in study and improve their learning performance. Experiential Learning Theory (ELT) provides a holistic model which emphasizes the central role that experience plays in the learning process. The most widely used learning theory is Kolb's experiential learning cycle [2-4] as shown in Fig. 1.

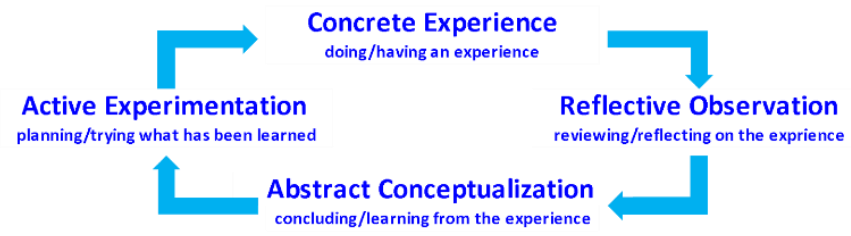

Fig. 1. Kolb's cycle of experiential learning.

In this paper, we present our exploration in applying the experiential learning in engineering education through the design and development of an exoskeleton [5] suit. Proposed in science fiction many decades ago, the exoskeleton is emerging from imagination to the real world nowadays with the advancing of key enabling technologies, and gradually found its role in many applications in medical, civilian and military. Moreover, an exoskeleton is an integration of many technologies spanning multiple engineering disciplinary. With this inspiration, we organized an 
undergraduate student team to work on the exoskeleton suit development. The suit is a studentcentered multifunctional platform that enables students in practicing diversified engineering design and explore innovative technologies. Through the development of the suit, participated engineering students explored and practiced technologies in various fields, such as CAD design and $3 \mathrm{D}$ printing, microprocessor and microcomputer system design, image processing, sensor and signal processing, wireless communication, etc. Through the development of the exoskeleton project, participated engineering students are strongly motivated and significantly promoted in engineering learning, practicing, exploring and innovating.

The rest of the paper is organized as follows: Sect. 2 provides an overview of the suit; Sect. 3 shows more details on design and development of core components in the suit; Sect.4 presents and discusses the educational aspect of the project, and finally Sect.5 concludes the whole work.

\section{Exoskeleton Suit Overview}

The development of the suit is a typical mechatronic project which integrates engineering designs in both mechanical and electrical. The electrical system diagram of the suit is shown in Fig. 2.

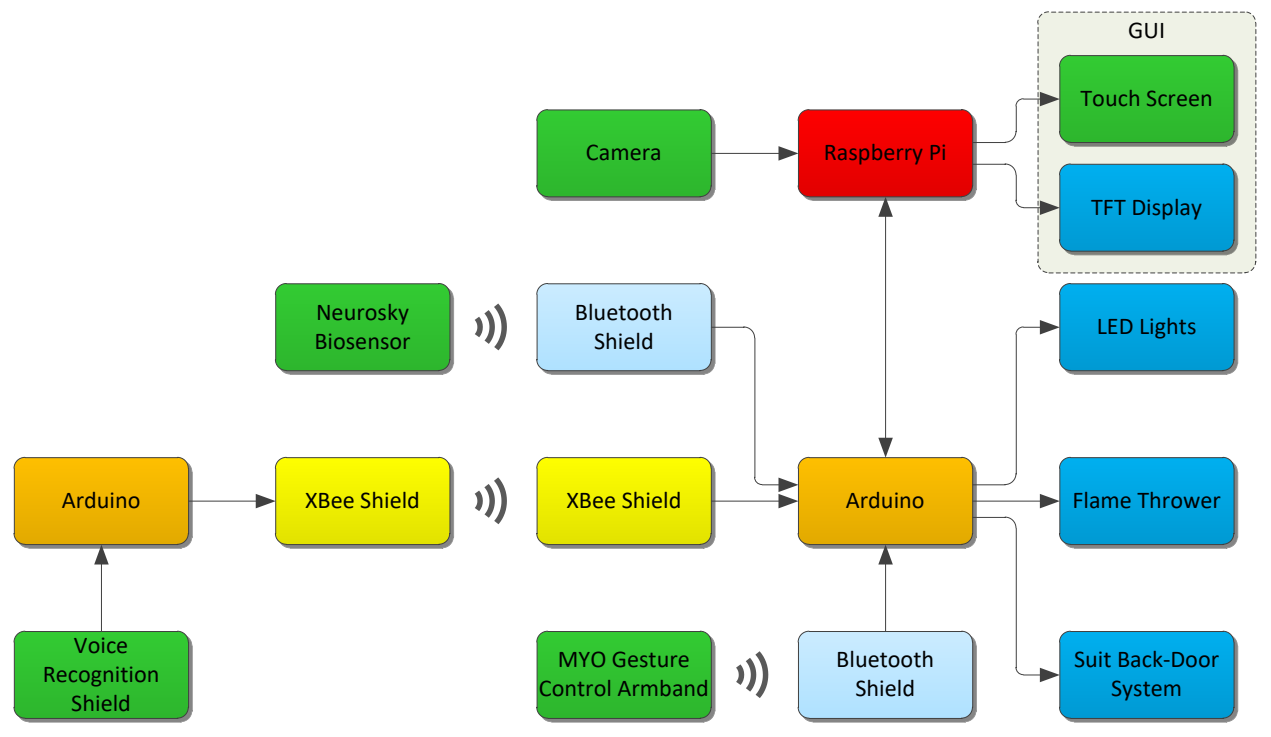

Fig. 2. Exoskeleton Suit System Diagram.

The basic functions of the suit are the following:

- Suit Structure

All external pieces and structural frame of the suit are designed in CAD software and then fabricated with a $3 \mathrm{D}$ printer. A back-door subsystem is integrated into the suit to enable the user's entrance to the suit easily.

1). External and base pieces

These built the internal structure and external part of the suit.

2). Backdoor system

The backdoor system enables the user to put on the entire suit from back easily. 
3). Other functional components

- Central Control Unit

The suit is controlled by the central control unit which is composed of a microcomputer Raspberry Pi 3 assisted with microprocessor Arduino MEGA. A Raspberry Pi 3 is a SingleBoard-Computer (SBC) equipped with an ARM v8 CPU running on 12.GHz. An Arduino MEGA is a microcontroller board with enriched $\mathrm{I} / \mathrm{O}$ pins/ports.

- User Interface

The suit provided diversified ways for the user to operate different functions embedded, including:

1). Touchscreen/LED display

The GUI for user input and operation of the suit.

2). Mind control

3). Gesture control

4). Voice control

- Imaging System

The imaging system is composed of the Raspberry Pi in the central control unit and the Pi camera(s). The imaging system enables advanced functions based on image processing algorithms, such as image enhancement, face recognition, night vision, etc.

- Power System

The suit is driven by a rechargeable battery. Battery usage is managed and monitored by the central control unit.

- Accessories

Because of the modularity design of the suit, there are many optional units with additional functions and features are available for users to choose and add onto the system conveniently, such as:

1). Fire Thrower

2). LED Lights

\section{Suit Development}

More details of design and development of components and functions integrated into the suit are provided as follows.

\subsection{External and Structural Design}

Fig. 3 demonstrates the design and fabrication process of a structural layer of the suit. First, based on the dimension of the user, plates for different positions are designed and drawn on cardboard, then they were used to cut out the fabric for connecting the plates. All the base and external pieces in the suit are designed in SolidWorks (Fig. 3(a)) and then fabricated by a 3D 
printer (Fig. 3(c)) with PLA ESun filament. All related plates are connected to the hinges in the backdoor system which can open and close automatically to enable the user put the suit on easily (Fig. 3(d)).

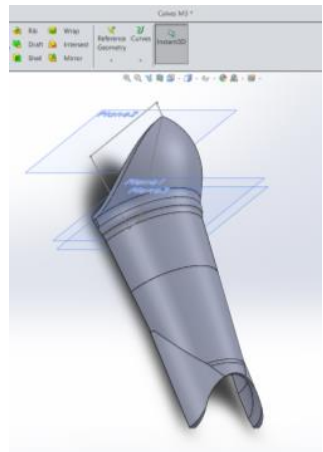

(a)

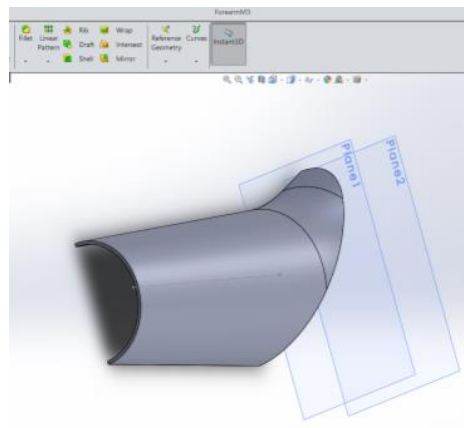

(b)

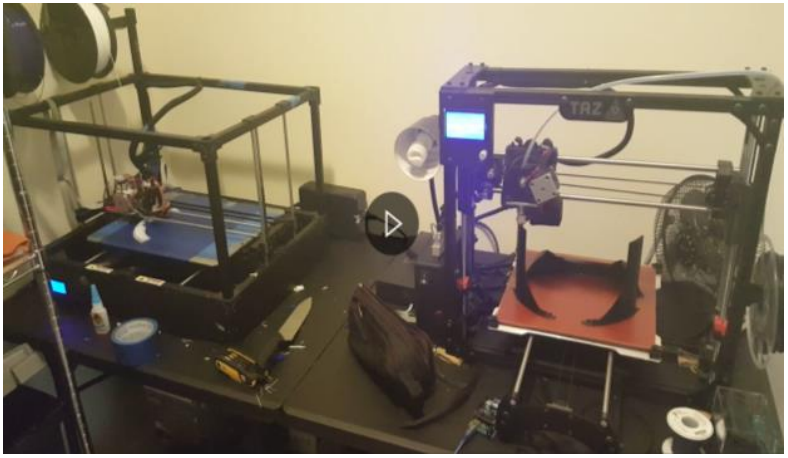

(c)

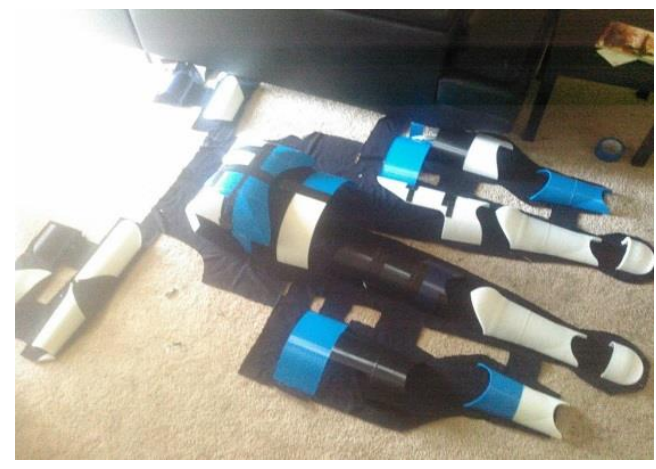

(d)

Fig. 3. (a), (b) Examples of CAD suit piece designs, (c) 3D printing the design, and (d) appearance of the raw suit.

\subsection{Central Control Unit}

A microcomputer Raspberry Pi 2 [6] is deployed to centrally control all functions of the suit, especially those advanced functions such as image processing, display and internet connection. Raspberry $\mathrm{Pi}$ is a low cost, credit-card sized computer with 1.2G Hz 64-bit quad-core ARM processor, which offers most functions of a desktop computer. In addition, one microprocessor Arduino Uno [7] is directly connected to the Pi for assisting basic functions because of its rich $\mathrm{I} / \mathrm{O}$ connections. And another Uno is used to support the wireless XBee communication between the voice recognition unit on the helmet and the central control unit on the body.

A Pi-TFT device is mounted on the Raspberry Pi for the graphic user interface (GUI) functions including display and touchscreen. The GUI is developed on Pi in Python language for displaying user the system information, receiving user commands to the suit and other advanced imaging/video functions.

\subsection{User Interface}

The suit has integrated many fancy interfacing devices for the user to customize the control of various functions. This also serves the experiential learning purpose for students to get exposed to different technologies. 


\subsubsection{Mind Control}

A bio-sensing device Neurosky [8] headset from Mindware is selected for the function of mind control of the suit. The headset is embedded in the helmet. It can sense the brainwaves to measure the levels of concentration, then wirelessly transfers the result to the central controller through BlueSmirf [9] the Bluetooth receiver. This feature can be used to activate/deactivate certain functions of the suit. In the current version, three level of user concentration (high/normal/low) is defined. Then LED lights on the suit in different colors and positions will be turned on corresponding to the level. This feature is shown in Fig. 4(a).

\subsubsection{Gesture Control}

Another bio-sensing device MYO [10] armband is selected for the function of gesture control of the suit. The MYO armband detects the user's muscle contractions and position, and can wirelessly transfer the data to the central control unit via Bluetooth module HM-11 [11]. In the current version, the gesture control is used to control the back-door system of the suit. This feature is shown in Fig. 4(b).

\subsubsection{Voice Control}

A Voice Recognition V3 [12] module is deployed for the function of voice control of the suit. It supports up to 80 different voice commands with maximum 7 voice commands working at the same time. Any sound could be trained as a command after the training to the kit. The voice recognition unit is embedded in the helmet, which can be detached from the rest of the suit without any hindrance. To serve this function, a pair of XBee modules and an additional Arduino are integrated into the helmet for the wireless communication. A voice command will be captured by the microphone, then processed and recognized by the V3 module, and transmitted to the central control unit through XBee modules. This feature is shown in Fig. 4(c).

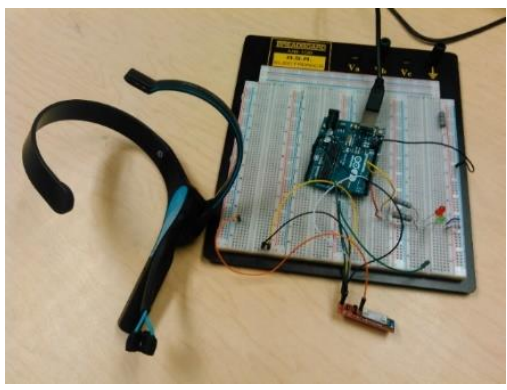

(a)

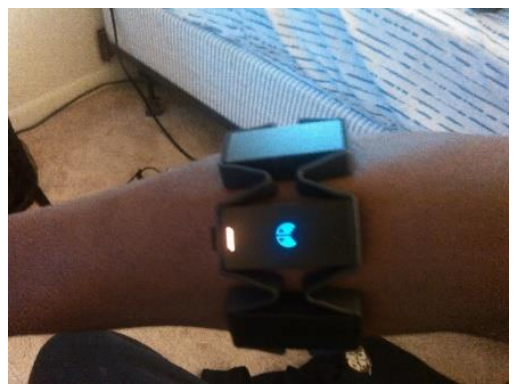

(b)

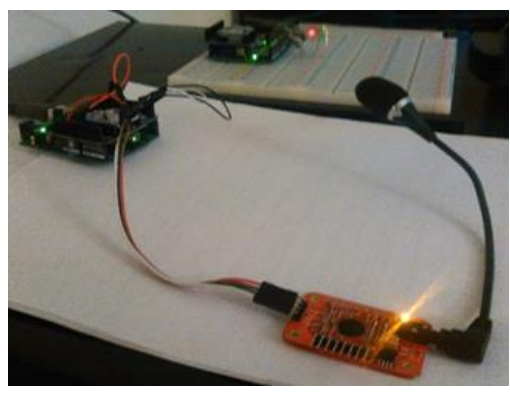

(c)

Fig. 4. Testing of different user interfacing devices: (a) Neurosky headset, (b) MYO armband, and (c) M3 voice recognition module.

\subsection{Imaging and Video Functions}

A Raspberry Pi camera module is used for imaging and video related functions. The camera is directed connect to the Raspberry Pi. Basic functions such as user controlled high-definition video recording and still photo capture are well supported by the device and can be easily achieved. Advanced functions, for example, face recognition and image enhancement can also be programmed and implemented in the Raspberry Pi. 


\subsection{Accessories}

Because of the modularity and open design of the suit, new functions and accessories can be easily developed and integrated into the system.

\subsubsection{Flamethrower:}

A palm flame thrower is designed and manufactured for the Suit as an optional accessory. A butane holder is designed in SolidWorks as shown in Fig. 5. It with an opening in the back where the bottom of the butane canister is located. This opening allows the butane holder to be pushed by an external force. The front end of the holder is thin enough for the nozzle to stick out and be wrapped with tubing. The tubing connects the butane nozzle into the flamethrower nozzle located in the palm of the flame glove. A waterproof digital servo is installed to push the butane through the canister and get ignited under the control of Arduino.

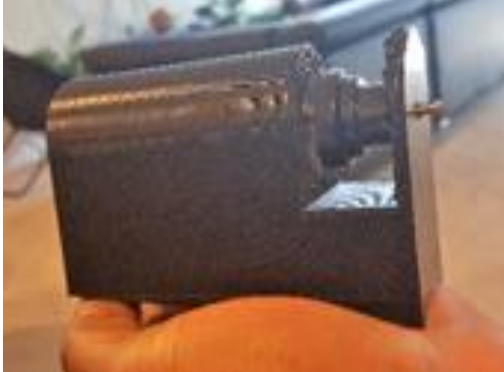

(a)

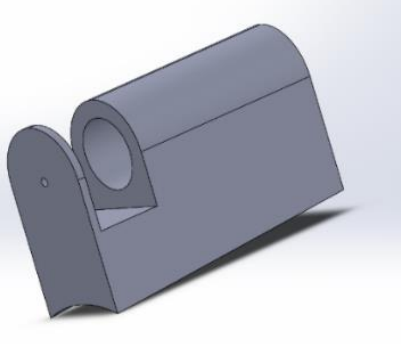

(b)

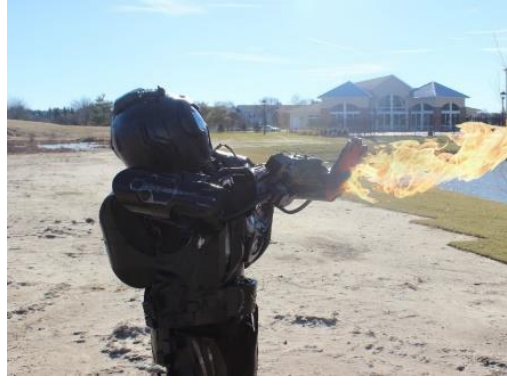

(c)

Fig. 5. The butane holder (a), its 3D CAD design (b), and operation (c).

\subsubsection{Lighting Subsystem}

Another optional accessory is the lighting subsystem. Fig. 6 shows the LED lights worked in different modes controlled by the brainwave signal collected by the Neurosky headset.
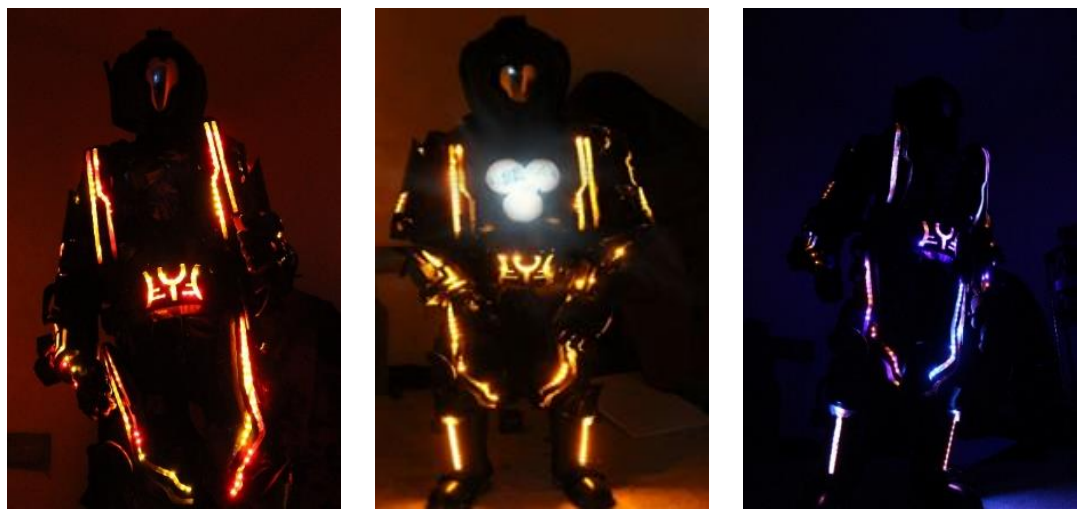

Fig. 6. Suit lighting system in different modes.

\subsubsection{Other Customizable Accessories}

As an open platform new modules can be easily developed and integrated to upgrade the suit, based on the teaching needs or student interest. As an example, Fig. 7 shows the design of a customized Pokemon style Charizard Arm designed and manufactured by engineering students. 
When the user gives the voice command "Charizard Go!" the signal will be captured by the voice recognition module to open its head and lunge jaw forward. Next, when the user pulls the hand back, it will be detected by sensors to trigger the shooting of orange and yellow fog from the palm up to 5 feet, to mimic the behavior of Pokemon Charizard.

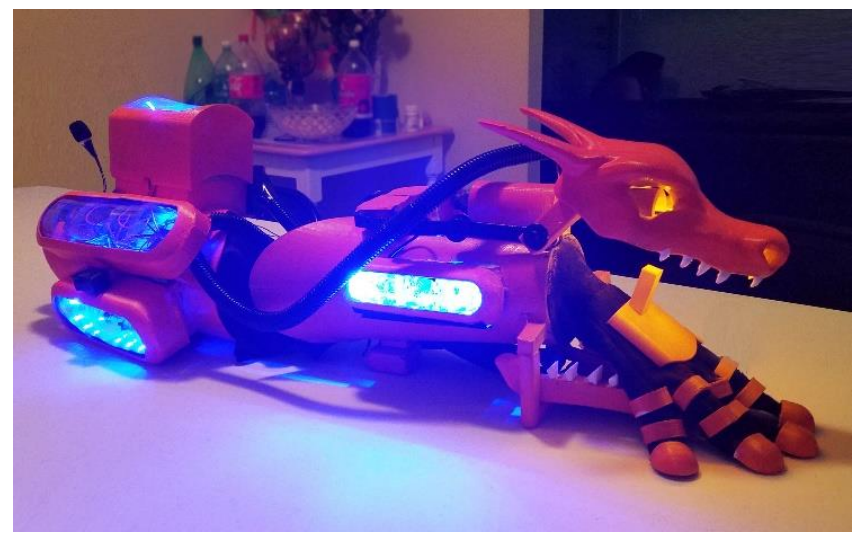

Fig. 7. Pokemon themed Charizard Arm for the suit

\section{Engineering Education through the Exoskeleton Suit Development}

The exoskeleton project at the beginning served as an undergraduate engineering student research project supported by NSF LSAMP program. As more and more features and functions were integrated into the suit, its value as an experiential learning tool has been recognized by the faculty mentor. And thus an interdisciplinary student team is created to concentrate on the development of the project through semesters. Various versions of the suit have been developed with different configurations and features, as shown in Fig. 8. The total cost of the latest one is around $\$ 1,500$, including all accessories.

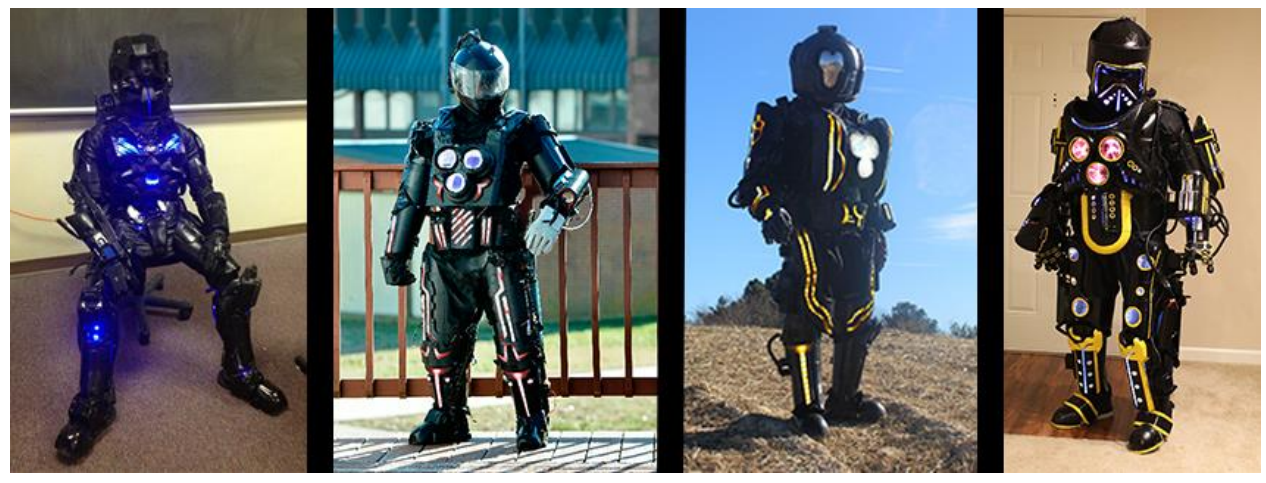

Fig. 8. Different phases of the exoskeleton suit.

\subsection{Features of the Exoskeleton Suit}

As an interdisciplinary engineering project platform, the exoskeleton suit possesses many outstanding properties. First is an attraction to students. Entertainment is an intrinsic feature of the suit which has attracted many students. Compare to other teaching tools, this suit is more interactive and user-friendly to students to develop new functions and modules and add-on to it, which made it a perfect tool to apply experiential learning for engineering curriculum. Moreover, 
the suit concept is more important to students than the existence of a fixed hardware itself. As a vivid example, students can receive the direct feeling how to build a complex project from scratch. Simple or complex, students are strongly encouraged to launch the building of their own suit by following the same way, or any other engineering project.

The second is the diversity of its functions. As mentioned above, in the current version of the suit, engineering topics involved span from CAD design and 3D printing to microprocessor and microcomputer system design, image processing, sensor and signal processing, wireless communication, etc. And more new functions can be easily integrated into the suit system.

Another important aspect involved in the suit development is the career development and entrepreneurship training for engineering students. With the upgrading to the suit and improving on features, its potential business value was recognized by the student. So far a few design patents of the suit have been approved. With the entrepreneurship training and incubating assistance provided by the university, a competitive business plan has been developed and implemented successfully by the key student developers. During the past a few years, they have received \$20,000 from Maryland Capital Enterprises (MCE), \$40,000 from the Eastern Shores Shore Hatchery Competition, and \$100,000 from Maryland Industrial Partnerships (MIPs).

\subsection{Integrating the Exoskeleton Suit Platform in Engineering Education}

The development of the exoskeleton suit and the integration the suit as a design platform have remarkably contributed to the engineering education in our department, in the following aspects.

First, through the development process of the suit, various functional modules have been identified, developed, documented and integrated into our Education Module Library (EML) [13], which greatly facilitates the learning of these modules for later students. The EML is a collection of typical engineering modules with diversified functions which can significantly lower down the difficulty for students in launching a new project and provide strong support during the whole implementation process. EML modules are developed by students, with the complete information of and details of how to build the functional module and integrate into a system step by step. During the exoskeleton suit project, many new EML modules are developed, including MYO armband, Neurosky headset, Voice Recognition V3 kit, etc. For engineering students who are interested in learning or implementing a similar function in their own projects, they can easily acquire technical support from the EML by accessing the responding modules developed for the suit.

The second is the utilization of the exoskeleton suit in engineering education as a powerful teaching tool. As mentioned above, many technologies have been adopted in the suit, such as microcomputer, CAD, signal processing, etc., which enabled the suit to be an intuitive courseware for in-class demonstration, analysis, and practice. For example, the instructor can present the source code to students in the classroom to show how it controls every component of the suit, and then modify the code and demonstrate the consequences. In another class, students can design a new piece in CAD software such as SolidWorks and print it out with a 3D printer to replace an external piece on the suit. These activities can bring students more impressive experiences than the explanation of abstract concepts and on-paper exercise, and will also greatly improve the teaching effectiveness. 
The last but not the least, the exoskeleton suit is a flexible platform to support student's engineering practice and exploration of new knowledge in different disciplines. Most functions on the suits are not fixed by can be updated, and the suit can be upgraded continuously to add more and more new functions. For example, with the on-suit Raspberry Pi and Pi-Camera, a student can develop and test new functions/algorithms in image processing, such as face recognition, object identification, etc. The Neurosky kit can be replaced with more professional devices for the student to study advanced signal processing algorithms for more precise mindcontrol. Shortly, most students can find their interests and define new projects on the suit platform. Right now, we are working on the development of OLED Augmentation Reality (AR) close-eye display for the suit which requires advanced image processing and cloud computing knowledge. Other engineering learning tools such as robotic arm and the unmanned vehicle are also pipelined be integrated to the suit through the student learning process.

\section{Conclusion}

In this paper, we present our practice in developing an exoskeleton suit as an experiential learning platform for students to develop interdisciplinary projects. The suit is a student-centered project that has integrated many functions in multiple disciplinaries. Engineering students in different levels and specializations can find their interest to practice engineering design and development based on the platform. And the suit also contributes to the entrepreneurship training to students and their success in business development. In future, we will keep developing and adding more learning modules onto the exoskeleton suit to facilitate students' experiential learning.

\section{References}

[1] X. Chen, "STEM Attrition: College Students' Paths into and out of STEM Fields. Statistical Analysis Report. NCES 2014-001," National Center for Education Statistics, 2013.

[2] D. A. Kolb, Experiential learning: Experience as the source of learning and development. Prentice-Hall Englewood Cliffs, NJ, 1984.

[3] R. Loo, "A meta-analytic examination of Kolb's learning style preferences among business majors," Journal of Education for Business, vol. 77, no. 5, pp. 252-256, 2002.

[4] D. Kolb, "Experiential learning as the science of learning and development," Englewood Cliffs NPH, editor1984, 1984.

[5] A. B. Zoss, H. Kazerooni, and A. Chu, "Biomechanical design of the Berkeley lower extremity exoskeleton (BLEEX)," IEEE/ASME Transactions On Mechatronics, vol. 11, no. 2, pp. 128-138, 2006.

[6] Raspberry Pi. Available: https://www.raspberrypi.org

[7] Arduino. Available: https://www.arduino.cc/

[8] Neurosky. Available: http://neurosky.com/

[9] Bluesmirf. Available: http://www.mouser.com/ProductDetail/SparkFun-Electronics/WRL12577/?qs=WyAARYrbSnbKwbjEv\%252bymNw\%3D\%3D\&gclid=CImD7vnsi9ICFYdWDQod EWkDJw

[10] MYO. Available: https://www.myo.com/

[11] HM-11. Available: http://wiki.seeed.cc/Bluetooth_V4.0_HM_11_BLE_Module/

[12] V3. Available: http://www.elechouse.com/elechouse/index.php?main_page=product_info\&cPath=\&products_id $=2254$ 
[13] L. Zhang, I. K. Dabipi, Y. Jin, and P. Matin, "Improving Undergraduate Engineering Education with Educational Module Library and Vertical Integration Projects," New Orleans, Louisiana, 2016/06/26, Available: https://peer.asee.org/25638 